\title{
A Multistep Extending Truncation Method towards Model Construction of Infinite-State Markov Chains
}

\author{
Kemin Wang, Yongbin Wang, Zhengtao Jiang, and Wenlong Fu \\ School of Computer Science, Communication University of China, Beijing 100024, China \\ Correspondence should be addressed to Kemin Wang; bbi-wkm@cuc.edu.cn
}

Received 9 October 2013; Accepted 14 December 2013; Published 12 January 2014

Academic Editor: Shuping He

Copyright (C) 2014 Kemin Wang et al. This is an open access article distributed under the Creative Commons Attribution License, which permits unrestricted use, distribution, and reproduction in any medium, provided the original work is properly cited.

The model checking of Infinite-State Continuous Time Markov Chains will inevitably encounter the state explosion problem when constructing the CTMCs model; our method is to get a truncated model of the infinite one; to get a sufficient truncated model to meet the model checking of Continuous Stochastic Logic based system properties, we propose a multistep extending advanced truncation method towards model construction of CTMCs and implement it in the INFAMY model checker; the experiment results show that our method is effective.

\section{Introduction}

Continuous Time Markov Chains (CTMCs) have been used in various areas of research as a formalism; so far, the model checking of CTMCs has been a hot research topic in computer science research communities. Some algorithms and implementations have been shown in several papers and tools [1-7]. However, our research aims to the model checking problem of the Infinite-State CTMCs, which means that the states of the CTMCs in our interest can be infinite. The papers [8-11] and the tool INFAMY [7] are with the same interest, which are also our research basis. Due to the explosion of states of CTMCs, our approach is based on a truncated CTMC model, which is determined by the exploration on the fly $[9,10]$, which means that the depth of model is computed dynamically by the exploration of states.

The truncation process is involved with reachability analysis $[9,10]$; that is, the transient probability is computed with the exploration of the model. The transient probability is carried out by uniformization method [10]. Together with the transient analysis when constructing the model, computation is very heavy, so to get a sufficient truncated model to meet the requirement of related Continuous Stochastic Logic (CSL) property or certain precision as fast as possible is our destination. We introduce a multistep extending advanced truncation method to meet this end, and the experimental results show our method is effective.
The main contents of the paper are organized as follows. Section 2 introduces the truncation based reachability analysis; Section 3 introduces an advanced truncation algorithm and do experiments. Section 4 proposes some multistep extending solutions and experiments. Section 5 talks about our result and next job.

\section{Truncation Based Reachability Analysis}

2.1. Finite Truncations. Let $\mathscr{C}=(S, \mathbf{R}, L)$ be a CTMC, where $S$ is a countable set of states, $\mathbf{R}:\left(S \times S \rightarrow \mathbb{R}_{\geq 0}\right)$ is the rate matrix, and $L: S \rightarrow 2^{A P}$ is a labeling function.

First, we introduce some paths and probabilistic measures from [9]. A (timed) infinite path is an infinite sequence $\sigma=$ $s_{1} t_{1} s_{2} t_{2} \ldots$ satisfying $\mathbf{R}\left(s_{i}, s_{i+1}\right)>0$, and $t_{i} \in \mathbb{R}_{\geq 0}$ for all $i=$ $1,2, \ldots$. For the path $\sigma$ and $i \in N$, let $\sigma[i]=s_{i}$ denote the $(i+1)$ th state, and let $\delta(\sigma, i)=t_{i}$ denote the time spent in $s_{i}$. For $t_{i} \in \mathbb{R}_{\geq 0}$, let $\sigma @ t$ denote $\sigma[i]$ such that $i$ is the smallest index with $t \leq \sum_{j=0}^{i} t_{j}$. For $\mathscr{C}$, let path $\mathscr{C}_{\infty}^{\mathscr{C}}$ denote the set of all paths, and let path $\mathrm{C}_{\infty}^{\mathscr{C}}(s)$ denote the set of all paths starting from $s$. For state $s \in S$, a probability measure, denoted by $\operatorname{Pr}_{s}^{\mathscr{C}}$, on the set $\operatorname{Pr}_{s}^{\mathscr{C}}(s)$ can be defined. A finite path is a finite sequence $\sigma=s_{1} t_{1} s_{2} t_{2} \ldots s_{k}$ for $k>0$ satisfying $\mathbf{R}\left(s_{i}, s_{i+1}\right)>0$ and $t_{i} \in \mathbb{R}_{\geq 0}$ for $i=1,2, \ldots, k-1$. Let len $(\sigma)=k-1$ denote the length of the path, first $(\sigma)=s_{1}$ denote the first state, and last $(\sigma)=s_{k}$ denote the last state of the path. Let path ${ }_{f}^{\mathscr{C}}$ denote 
the set of all finite paths. We omit the superscript $\mathscr{C}$ if it is clear from context.

Next, we introduce the notion of depth. Let $S_{0}$ be a finite subset of $S$ with depth 0 ; that is, $d(s)=0$ for $s \in S_{0}$. For now, one may think of $S_{0}$ as being equal to the support of the initial distribution $\mu$. However, $S_{0}$ can be an arbitrary finite set. This will allow us not only to deal with the initial distribution, but also to compute truncation depths for nested CSL formulas. The depth of state $s$ corresponds to the minimal distance from the set $S_{0}$.

Definition 1. For $\mathscr{C}$ and $S_{0} \subset S$, the depth function $d: S \rightarrow \mathbb{N}$ is defined by $d_{S_{0}}(s)=\min \left\{\operatorname{len}(\sigma) \mid \sigma \in \operatorname{path}_{f} \wedge \operatorname{first}(\sigma) \epsilon\right.$ $\left.S_{0} \wedge \operatorname{last}(\sigma)=s\right\}$.

Observe that $d_{S_{0}}(s)=0$ for all $s \in S_{0}$. The subscript is omitted if $S_{0}$ is clear from the context. Intuitively, $d(s)$ corresponds to the minimal length of any finite path starting from $S_{0}$ and ending in $s$.

We consider a partition of the state space $S=\bigcup_{i \in \mathbb{N}} S_{i}$, where $S_{i}:=\{s \in S \mid d(s)=i\}$ is the set of states with depth $i$. We say that the set $S_{i}$ is the layer with depth $i$ and call its elements layer- $i$ states. Assume that $\perp \in S$ is a special state not in $S$ and furthermore $\perp$ is also an atomic proposition not in $A P$. For $k \in \mathbb{N}$, let $S_{>k} \subset S$ denote the set of states with depth greater than $k$; that is, $S_{>k}=\{s \mid d(s)>k\}$.

Further, we write $S_{\leq k}=S \backslash S_{>k}$. We define the $k$-truncated CTMC as follows.

Definition 2. Let $\mathscr{C}=(S, \mathbf{R}, L)$ be a CTMC and let $S_{0}$ be the layer-zero states. For $k \in \mathbb{N}$, we define the $k$-truncated CTMC of $\mathscr{C}$ by $\left.\mathscr{C}\right|_{k}=\left(\left.S\right|_{k}, \mathbf{R}_{k}, L_{k}\right)$, where the states are $\left.S\right|_{k}=S_{\leq k} \cup\{\perp\}$. The labeling function $L_{k}:\left.S\right|_{k} \rightarrow A P \cup\{\perp\}$ is defined by $L_{k}(s)=L(s)$ if $s \in S_{\leq k}$, and $L_{k}(\perp)=\{\perp\}$. The rate matrix is defined by the following: $\mathbf{R}_{k}\left(s, s^{\prime}\right)$ equals $S\left(s, s^{\prime}\right)$ if $s, s^{\prime} \in S_{\leq k}$, equals $\mathbf{R}\left(s, S_{>k}\right)=\sum_{t \in S_{>k}} \mathbf{R}(s, t)$ if $s \in S_{k}, s^{\prime}=\perp$, and equals 0 otherwise.

The $k$-truncation of an infinite CTMC is illustrated in Figure 1. Intuitively, the transition matrix is restricted to the truncated state space $\left.S\right|_{k}$, and $\perp$ is the distinguished absorbing state, which, by construction, is only reachable from states with depth $k$. In state $\perp$ only the atomic proposition $\perp$ holds, which indicates that the system is in state $\perp$. Since we consider finitely branching CTMCs, not surprisingly, the $k$-truncated CTMC $\left.\mathscr{C}\right|_{k}$ is always finite. The absorbing state $\perp$ has been introduced to abstract $S_{>k}$. We assume that $\perp \notin \operatorname{Sat}(\Phi)$ for any state formula $\Phi$. We consider the probability of reaching the absorbing state $\perp$ in the $k$-truncated CTMC $\left.\mathscr{C}\right|_{k}$, that is, $\left.\vec{\pi}^{\mathscr{C}}\right|_{k}(s, t)(\perp)$. For mere notational convenience, we extend $\vec{\pi}^{\left.\mathscr{C}\right|_{k}}$ to states of $\mathscr{C}$ with depth higher than $k: \vec{\pi}^{\left.\mathscr{C}\right|_{k}}(s, t)\left(s^{\prime}\right)=0$ for all $s^{\prime} \in S$ with $d\left(s^{\prime}\right)>k$. For a fixed $k$, we define the forward rate $\operatorname{fr}_{k}(s)$ of a state $s \in S_{\leq k}$ within $\left.\mathscr{C}\right|_{k}$. For $s \in S_{\leq k} \backslash S_{k}$, it is the sum of the rates that go into the next layer $\operatorname{fr}_{k}(s)=\mathbf{R}\left(s, S_{d(s)+1}\right)$, and, for $s \in S_{k}$, it is the sum $\operatorname{fr}_{k}(s)=\mathbf{R}\left(s, S_{>k}\right)$ of the rates entering states in $S_{k}$.

2.2. The Logic CSL. The logic we consider is CSL without steady-state operator and unbounded until operator [10]. Let

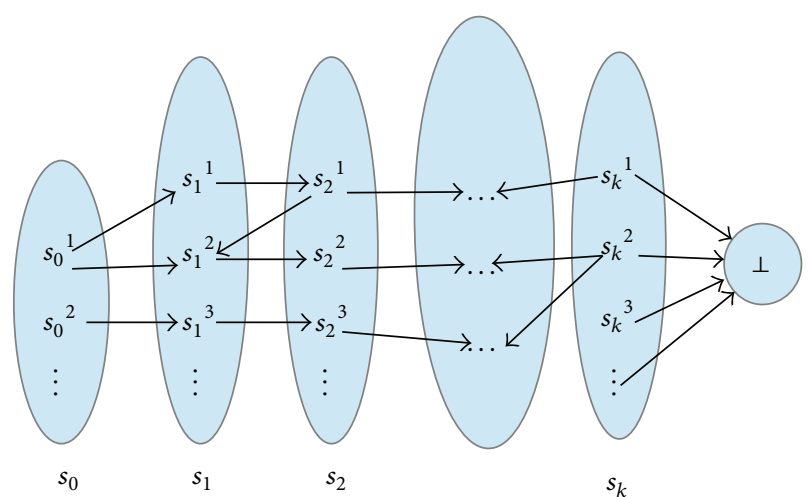

Figure 1: A $k$-depth truncated CTMC.

$I=\left[t, t^{\prime}\right]$ be an interval with $t, t^{\prime} \in \mathbb{R}_{\geq 0}$ and $t \leq t^{\prime}$. Let $p \in[0,1]$ and $\unlhd \in\{\leq,<,>, \geq\}$. The syntax of state formula $\Phi$ and path formulas $\phi$ is

$$
\begin{gathered}
\Phi=a|\neg \Phi| \Phi \wedge \Phi \mid \mathscr{P}_{\unlhd p}(\phi), \\
\phi=\mathscr{X}^{I} \Phi \mid \Phi \mathcal{U}^{I} \Phi .
\end{gathered}
$$

The semantics of the state formulas and path formula are precisely defined in [10]. In this paper, the state formula like the kind of $\mathscr{P}_{\unlhd p}(\phi)$ will be more focused on; it means that starting some state, the probability of the system that satisfies a path formula $\phi$ would meet the rational relation $\unlhd p$ or not, so the first solution to this problem is to compute this probability, so this is reduced to transient analysis, which is our main interest, to improve the efficiency of transient analysis for the whole model while extending the border states layer by layer.

2.3. Transient Analysis. Transient analysis means that, starting at state $s$, the transient probability vector at time $t$, it can be denoted as $\vec{\pi}(s, t)$. If $t=0$, we have $\vec{\pi}(s, 0)\left(s^{\prime}\right)=$ 1 if $s=s^{\prime}$ and 0 otherwise. The uniformization method based solution of $\vec{\pi}(s, t)$ was given at [10]. Let $t>0$ and $\vec{\pi}(s, t)=\sum_{i=0}^{\infty} \varphi(i, q t) \vec{\pi}(s, i)$, where $q$ is the uniformization rate satisfying $q \geq \sup _{s \in S} \mathbf{R}(s, S)$ and $\varphi(i, q t)=e^{-q t}\left((q t)^{i} / i !\right)$ denotes the $i$ th Poisson probability with parameter $q t$ and vector $\vec{\pi}(s, i)$ is transient probability of the uniformized DTMC uni $(\mathscr{C})$ at step $i$.

The truncated model in our method is given by dynamically exploring from the initial state(s); once we want to add a layer on it, we need to compute the reachability probability from the initial(s) to the current layer states which we want to add. As shown in Figure 1, $s_{0}$ is the initial states set, and each of $s_{1}, s_{2}, \ldots, s_{k}$ is explored dynamically, so we get a $k$ depth truncated model of the infinite one. $\perp$ is the absorbing state, which is the border states set, for which the sum of the reachability probabilities from the initial state(s) is less than $\varepsilon$; $\varepsilon$ is the precision of the result, which can be $10^{-6}, 10^{-9}$, or $10^{-12}$, and so forth, which can be set under INFAMY model checker as a circumstance variable for some certain need.

The reachability probability is carried out by the uniformization method to the CTMC, which is a relatively fast 


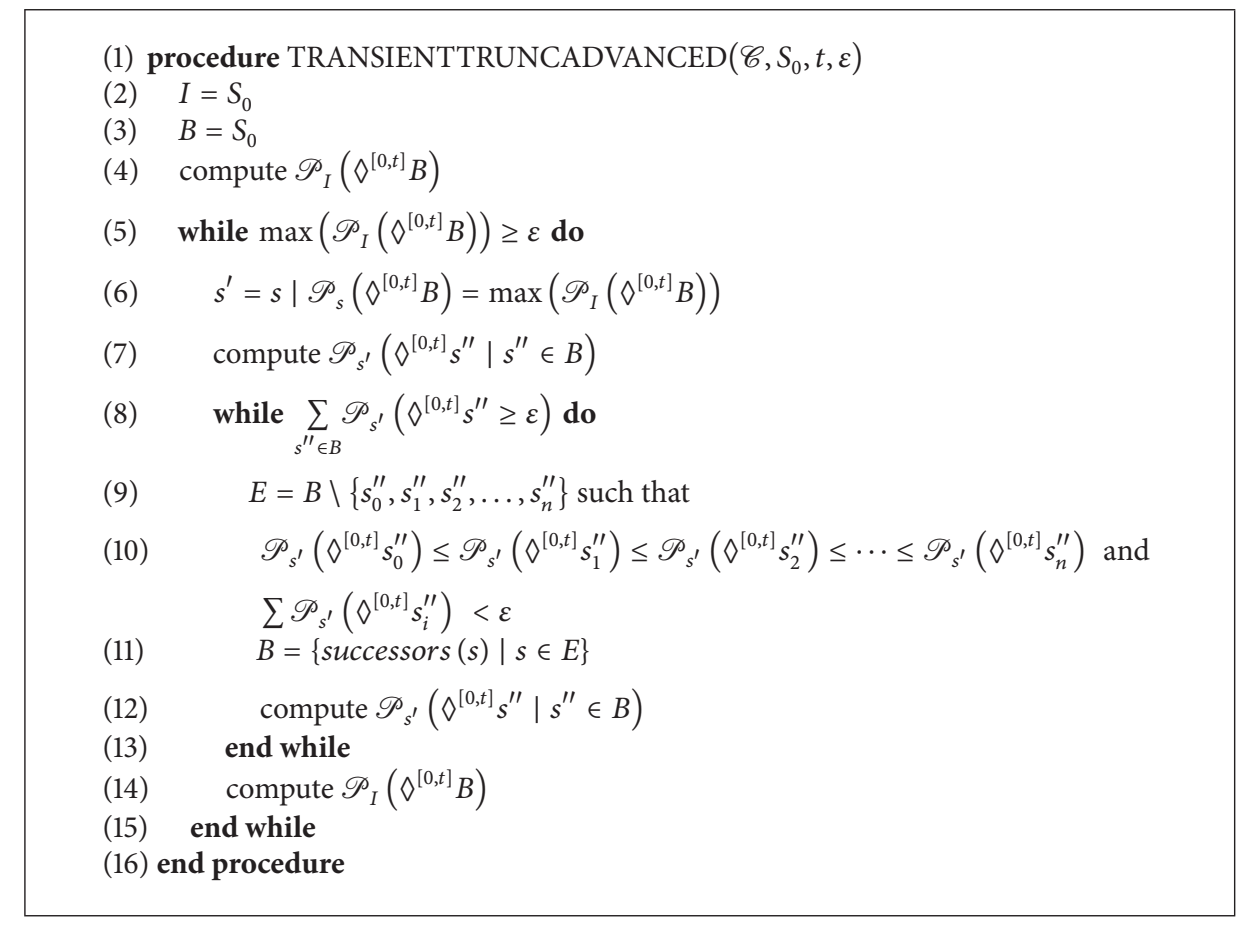

Algorithm 1: An advanced truncation algorithm towards construction of CTMC.

method to get the transient probability at a certain bound time $t$ at some state, which is denoted as $\mathscr{P}\left(\diamond^{[0, t]} s\right)$. However, if the state explosion situation is very serious, the time to construct the model layer by layer would be of much cost. So we introduce an advanced truncation algorithm to explore the states, this method can improve the efficiency of the model constructing and model checking, and then at Section 4, we further propose some multistep extending solutions, which are implemented based on INFAMY model checker, the experiment results show that these solutions can help to improve the efficiency.

\section{An Advanced Truncation Algorithm and Experiments}

The truncation process is implemented by extending the states layer by layer from the initial states, and all the new states need to be transient analyzed and then to be extended further no matter how small the probability is. For that the precision of the result is under some certain value, so some states with relatively nearly no contribution to the result can be omitted when extending; thus, an advanced truncation algorithm is introduced. It is different from the finite state projection (FSP) $[9,10]$ and layered chain and uniform chain method $[9,10]$. The algorithm is shown in Algorithm 1.

The algorithm aims to stop the extending of less important (small probability) states and proceed to the extending of much important (large probability) states; the less or more is determined by the state reduction policy; as shown in Figure 2, line 7, $\mathscr{P}_{s^{\prime}}\left(\nabla^{[0, t]} s_{0}^{\prime \prime}\right) \leq \mathscr{P}_{s^{\prime}}\left(\diamond^{[0, t]} s_{1}^{\prime \prime}\right) \leq \mathscr{P}_{s^{\prime}}\left(\diamond^{[0, t]} s_{2}^{\prime \prime}\right) \leq$ $\cdots \leq \mathscr{P}_{s^{\prime}}\left(\diamond^{[0, t]} s_{n}^{\prime \prime}\right)$ and $\sum \mathscr{P}_{s^{\prime}}\left(\diamond^{[0, t]} s_{i}^{\prime \prime}\right)<\varepsilon$. The policy means that the states in the border states set, which has been sorted upward, $s_{0}^{\prime \prime}, s_{1}^{\prime \prime}, s_{2}^{\prime \prime}, \ldots, s_{n}^{\prime \prime}$, will be excluded from the extending states set, for the sum of them is just exactly less than the precision. So with this policy, the number of states to be transient analyzed will be smaller to the FSP and forwardlayered based model.

We consider the dependability of a fault-tolerant workstation cluster which is directly taken from case studies of [7]. Figure 2 depicts a dependable cluster of workstations. The cluster consists of two subclusters, which, in turn, contain $N$ workstations connected via a central switch. The two switches are connected via a backbone. Each component of the system can break down and is then fixed by a single repair unit responsible for the entire system. Hereby, the quality of service (QoS) constraint minimum requires at least $k(k<$ $N)$ workstations to be operational, where $k=\lfloor(3 / 4) N\rfloor$. Workstations have to be connected via switches. If in each subcluster the number of operational workstations is smaller than $k$, the backbone is required to be operational to provide the required service. We consider the property.

$\mathscr{P}=? \diamond[0, t] \neg$ Minimum. This probability means that the QoS drops below minimum quality within $t$ time unit.

For the property, we compare PRISM [6], FSP method and layered method of INFAMY. The results are given in Tables 1, 2, and 3. Because the resulting probabilities are very small in some cases, we use a precision of $10^{-6}$ here, for the computation of the truncation point. Results for INFAMY are given for the layered chain, FSP, and advanced configurations, respectively. The uniform chain configuration is omitted, as it is always dominated by the layered chain configuration. PRISM implements three different engines: a sparse-matrix and two symbolic engines. We used the sparse-matrix engine 
TABLE 1: Experiments of forward-layered method of INFAMY.

\begin{tabular}{|c|c|c|c|c|}
\hline \multirow{2}{*}{ Time bound $t$} & \multicolumn{4}{|c|}{ Forward-layered } \\
\hline & Depth & Time (s) & $n$ & Prob \\
\hline 5.0 & 316 & 450 & 1668363 & {$[0.9 E-06,1.50 E-06]$} \\
\hline 10.0 & 517 & 661.5 & 2373652 & {$[3.2 E-06,3.8 E-06]$} \\
\hline 20.0 & 517 & 1293 & 2373652 & {$[8.8 E-06,9.4 E-06]$} \\
\hline 30.0 & 517 & 1453 & 2373652 & {$[1.45 E-05,1.51 E-05]$} \\
\hline 50.0 & 517 & 2096 & 2373652 & {$[2.61 E-05,2.67 E-05]$} \\
\hline
\end{tabular}

TABLE 2: Experiments of advanced method of INFAMY.

\begin{tabular}{lcccc}
\hline Time bound $t$ & & \multicolumn{2}{c}{ Advanced } & Prob \\
\hline 5.0 & Depth & Time $(\mathrm{s})$ & 4071 & {$[0.9 E-06,1.50 E-06]$} \\
10.0 & 18 & 6.6 & 7118 & {$[3.2 E-06,3.9 E-06]$} \\
20.0 & 25 & 23.3 & 14434 & {$[8.8 E-06,9.5 E-06]$} \\
30.0 & 38 & 105.2 & 22426 & {$[1.45 E-05,1.52 E-05]$} \\
50.0 & 50 & 271.6 & 38448 & {$[2.61 E-05,2.68 E-05]$} \\
\hline
\end{tabular}

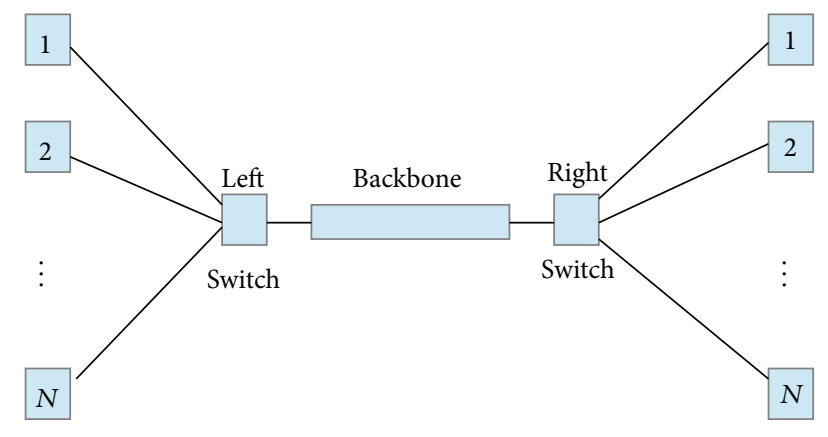

Figure 2: Dependable clusters of workstations.

as it was the fastest one. The results are shown in Tables 1, 2, and 3.

The experiment conditions are shown as follows.

Host Machine: it includes Mac Book, OS: Mac OS X 10.6.8, Processor: $2.2 \mathrm{GHz}$ Intel Core 2 Duo T7500, and Storage: $2 \mathrm{~GB} 667 \mathrm{MHz}$ DDR3 SDRAM.

Guest Machine: it includes Virtual Machine Software: VirtualBox 4.1 .14 r77440 for mac; Virtual OS: Linux ubuntu12.04 LTS 32Bit; Processor: 2.2 GHz Intel Core 2 Duo T7500; Storage: $512 \mathrm{MB}$

From Tables 1, 2, and 3, we can see that, for $t \leq 20$, FSP based INFAMY is faster, but for $t \leq 30, t \leq 50$, INFAMY model checker needs more time; this is because the transient analysis when constructing the model needs more computing. This is also the result of [11]. For the advanced method of the context, as in Tables 1, 2, and 3, comparing advanced with FSP, we can get that, under advanced based method, the depth of model is much deeper, and the states number is smaller; for the time costing, for $t \leq 20, t \leq 30$, the costing is less reduced, but for $t \leq$ 50 , the costing is greater; this result is reasonable, for the exploration policy is essentially undeterministically efficient for different models; for the current case, when $t \leq 50$, the current states number is very large, even with the reduction policy, with no contribution for it any more. We need to take other techniques to tackle this situation. Thus, we propose a multistep extending solution. See Section 4.

\section{Multistep Extending Solutions and Experiments}

The mutistep extending solution aims to reduce the extending of less important (small probability) states and enhance the extending of more important (large probability) states; for the latter states, we can, for example, extend two or more steps per extending, and for the former states, we can, for example, extend one step per extending, as we know that transient probabilities will be computed once again before the states were added to the border states, so, if we extend two or more steps, transient analysis at the intermediate states will be omitted; thus, time on the model construction will be reduced; then we can make the model much faster to converge to the absorbing state.

We continue with the upper case study. As shown in Algorithm 1, Line 11, $B=\{\operatorname{successors}(s) \mid s \in E\}$ is to extend the model from set $E$ and get the border states set $B$. This means that the advanced method in the upper section is extending one step per extending. Now, we design some multistep extending solutions:

(1) two-step extending solution, that is, $B=$ $\{\operatorname{successors}(\operatorname{successors}(s)) \mid s \in E\}$;

(2) three-step extending solution, that is, $B=$ $\{$ successors $($ successors $($ successors $(s))) \mid s \in E\}$;

(3) synthesis solution 1: a synthesis extending solution separates set $E$ to three parts, as set $E$ is a sorted set, which is sorted upward by the probabilities, so we can separate $E$ as $E_{1}, E_{2}$, and $E_{3}$, and the sizes of each part are the same. Then we can get 
TABLE 3: Experiments of PRISM and FSP method of INFAMY.

\begin{tabular}{|c|c|c|c|c|c|c|}
\hline \multirow{2}{*}{ Time bound $t$} & \multicolumn{2}{|c|}{ PRISM (sparse engine) } & \multicolumn{4}{|c|}{ FSP } \\
\hline & $\begin{array}{c}\text { Time (s) } \\
\text { (construct/M.C.ing) }\end{array}$ & Prob & Depth & Time (s) & $n$ & Prob \\
\hline 5.0 & $1.868 / 46.5$ & $1.16 E-06$ & 16 & 5.7 & 4405 & {$[0.9 E-06,1.70 E-06]$} \\
\hline 10.0 & $1.8 / 77.265$ & $3.51 E-06$ & 22 & 20.7 & 8425 & {$[3.2 E-06,4.0 E-06]$} \\
\hline 20.0 & $1.8 / 127.072$ & $9.06 E-06$ & 33 & 113.9 & 19161 & {$[8.8 E-06,9.7 E-06]$} \\
\hline 30.0 & $1.8 / 179.675$ & $1.48 E-05$ & 42 & 353.6 & 31185 & {$[1.45 E-05,1.56 E-05]$} \\
\hline 50.0 & $1.8 / 274.593$ & $2.64 E-05$ & 57 & 1219.7 & 57705 & {$[2.61 E-05,2.73 E-05]$} \\
\hline
\end{tabular}

TABLE 4: Experiment data under different extending solutions.

\begin{tabular}{|c|c|c|c|c|c|c|c|c|c|}
\hline \multirow{2}{*}{ Time bound $t$} & \multicolumn{3}{|c|}{ Advanced (one step per extending) } & \multicolumn{3}{|c|}{ Two-step per extending } & \multicolumn{3}{|c|}{ Three-step per extending } \\
\hline & Depth & Time (s) & n & Depth & Time (s) & $n$ & Depth & Time (s) & $n$ \\
\hline 5.0 & 18 & 6.6 & 4071 & 20 & 8.9 & 4756 & 33 & 15.0 & 7895 \\
\hline 10.0 & 25 & 23.3 & 7118 & 29 & 26.5 & 8027 & 38 & 41.3 & 11849 \\
\hline 20.0 & 38 & 105.2 & 14434 & 42 & 115.3 & 16315 & 52 & 174.0 & 20816 \\
\hline 30.0 & 50 & 271.6 & 22426 & 54 & 327.6 & 25308 & 64 & 456.0 & 30785 \\
\hline 50.0 & 69 & 1491.5 & 38448 & 72 & 1006.5 & 43824 & 82 & 1572.6 & 50942 \\
\hline$t$ & \multicolumn{3}{|c|}{ Synthesis solution 1} & \multicolumn{3}{|c|}{ Synthesis solution 2} & \multicolumn{3}{|c|}{ Solution D } \\
\hline 5.0 & 35 & 11.9 & 6539 & 20 & 9.6 & 4564 & 20 & 8.6 & 4459 \\
\hline 10.0 & 42 & 35.4 & 9772 & 26 & 29.7 & 7646 & 26 & 24.9 & 7383 \\
\hline 20.0 & 53 & 131.2 & 17777 & 41 & 111.7 & 15621 & 39 & 91.1 & 15170 \\
\hline 30.0 & 61 & 367.4 & 26362 & 53 & 279.7 & 24239 & 50 & 250.9 & 23063 \\
\hline 50.0 & 74 & 1349.6 & 45170 & 71 & 989.1 & 42703 & 71 & 791 & 40403 \\
\hline
\end{tabular}

(i) $B_{1}=\left\{\operatorname{successors}(s) \mid s \in E_{1}\right\}$;

(ii) $B_{2}=\left\{\right.$ successors $($ successors $\left.(s)) \mid s \in E_{2}\right\}$;

(iii) $B_{3}=\{$ successors $($ successors $($ successors $(s))) \mid$ $\left.s \in E_{3}\right\}$;

(iv) $B=B_{1} \cup B_{2} \cup B_{3}$;

(4) synthesis solution 2 separates set $E$ to two parts, so we can separate $E$ to $E_{1}$ and $E_{2}$, and the sizes of the two parts are the same. Then we can get
(i) $B_{1}=\left\{\operatorname{successors}(s) \mid s \in E_{1}\right\}$;
(ii) $B_{2}=\left\{\right.$ successors $($ successors $\left.(s)) \mid s \in E_{2}\right\}$;
(iii) $B=B_{1} \cup B_{2}$.

The experiments data are shown in Table 4. From the data in Table 4, we can see that under different extending solutions, the times needed are different, synthesis solution 2 performs better, when $t \leq 50$, and the time (including model constructing and model checking) has been reduced to $1000 \mathrm{~s}$. This means that for this case, the step per extending should be relatively small; for synthesis solution 2, half small states extend one step per-extending, and other half large states extend two steps per extending. And for this, we continue to propose a solution to revise synthesis solution 2, named Solution $\mathrm{D}$ the policy is as follows: For the states $e_{0}, e_{1}, \ldots, e_{n}$ in set $E$, the gap between the largest and smallest probabilities: $\operatorname{ProbGap}=\operatorname{Prob}\left(E_{n}\right)-\operatorname{Prob}\left(E_{0}\right)$. We can separate set $E$ to two parts $E_{1}$ and $E_{2}$, such that. (i) $E_{1}=\left\{e_{0}, e_{1}, \ldots, e_{i} \mid e_{i} \leq \operatorname{Prob}\left(e_{0}+\operatorname{ProbGap} / 2\right)\right.$ and $\left.e_{i+1}>\operatorname{Prob}\left(e_{0}+\operatorname{ProbGap} / 2\right)\right\}$

(ii) $E_{2}=E \backslash E_{1}$.

And then we can get that

(i) $B_{1}=\left\{\operatorname{successors}(s) \mid s \in E_{1}\right\}$;

(ii) $B_{2}=\left\{\right.$ successors $($ successors $\left.(s)) \mid s \in E_{2}\right\}$;

(iii) $B=B_{1} \cup B_{2}$.

The experiment results are as shown in Table 4. From Table 4, we can see that current solution $\mathrm{D}$ performs better on this case.

\section{Conclusion}

The multistep extending advanced truncation method can improve the efficiency of model construction of Infinite-State CTMCs; this is because the transient probabilities of states which have been jumped have not been computed, so to some extent this method is effective; however, which solution performs better needs to be experimented; there is no general solution that fits well for all cases. The efficiency is determined by the iterations when computing the transient probability. Less iteration is more efficient. However, this approach is essentially a linear approach to improve the efficiency; when the outsider state gets explosion, this approach will be less effective; just as in our case study, this approach can be used effectively to improve the model checking efficiency at a relatively small time bound $t$. For future work, we need 
to consider other techniques to tackle the state explosion problem on model checking of CTMCs. And other works like [12-15] can also be considered.

\section{Conflict of Interests}

The authors declare that there is no conflict of interests regarding the publication of this paper.

\section{Acknowledgments}

The authors would like to thank Lijun Zhang and Ernst Moritz Hahn for their original work on INFAMY model checker and the free contribution of the tool codes; Definitions 1 and 2 are directly taken from [10]. This paper is partially funded by the Communication University of China, 2013 Planned Research Program, and Research Funding Foundation of School of Computer Science, and the scholarship from China Scholarship Council for the Excellent University Teachers Study Abroad Program and supported by the National Natural Science Foundation of China (61103199), Beijing Municipal Natural Science Foundation (4112052); Supported by Engineering Program Project of CUC (3132013XNG1326, 3132013XNG1321), and the National Key Science and Technology Pillar Programs of China (2012BAH51F02, 2012BAH38F05, and 2013BAH66F02).

\section{References}

[1] A. Aziz, K. Sanwal, V. Singhal, and R. Brayton, Verifying Continuous Time Markov Chains, pp. 269-276, Springer, 1996.

[2] C. Baier, J. P. Katoen, and H. Hermanns, "Approximative symbolic model checking of continuous-time markov chains," in CONCUR '99 Concurrency Theory, vol. 1664 of Lecture Notes in Computer Science, pp. 146-161, Springer, 1999.

[3] H. Hermanns, J. Meyer-Kayser, and M. Siegle, "Multi terminal binary decision diagrams to represent and analyse continuous time markov chains," in Proceedings of the 3rd International Workshop on the Numerical Solution of Markov Chains, pp. 188207, Citeseer, 1999.

[4] J. P. Katoen, M. Kwiatkowska, G. Norman, and D. Parker, Faster and Symbolic CTMC Model Checking, Springer, 2001.

[5] C. Baier, B. Haverkort, H. Hermanns, and J. Katoen, "Modelchecking algorithms for continuous-time Markov chains," IEEE Transactions on Software Engineering, vol. 29, no. 6, pp. 524-541, 2003.

[6] Prism model checker, http://www.prismmodelchecker.org/.

[7] Infamy model checker, http://depend.cs.uni-sb.de/tools/infamy/.

[8] A. Remke, B. R. Haverkort, and L. Cloth, "Model checking infinite-state Markov chains," in Proceedings of the 11th International Conference on Tools and Algorithms for the Construction and Analysis of Systems (TACAS '05), vol. 3440 of Lecture Notes in Computer Science, pp. 237-252, Springer, April 2005.

[9] E. M. Hahn, H. Hermanns, B. Wachter, and L. Zhang, “Timebounded model checking of infinite-state continuous-time markov chains," Fundamenta Informaticae, vol. 95, no. 1, pp. 129-155, 2009.

[10] L. Zhang, H. Hermanns, E. M. Hahn, and B. Wachter, “Timebounded model checking of infinite-state continuous-time markov chains," in Proceedings of the 8th International Conference on Application of Concurrency to System Design (ACSD '08), pp. 98-107, June 2008.

[11] E. M. Hahn, H. Hermanns, B. Wachter, and L. Zhang, "Infamy: an infinite-state markov model checker," in Computer Aided Verification, Springer, pp. 641-647, 2009.

[12] S. He and F. Liu, "Robust stabilization of stochastic Markovian jumping systems via proportional-integral control," Signal Processing, vol. 91, no. 11, pp. 2478-2486, 2011.

[13] S. He and F. Liu, "Robust $l_{2}-l_{\infty}$ filtering of time-delay jump systems with respect to the finite-time interval," Mathematical Problems in Engineering, vol. 2011, Article ID 839648, 17 pages, 2011.

[14] S. He, Z. Ding, and F. Liu, "Output regulation of a class of continuous-time markovian jumping systems," Signal Processing, vol. 93, no. 2, pp. 411-419, 2013.

[15] H. Shen, S. Xu, J. Lu, and J. Zhou, "Passivity-based control for uncertain stochastic jumping systems with mode-dependent round-trip time delays," Journal of the Franklin Institute, vol. 349, no. 5, pp. 1665-1680, 2012. 


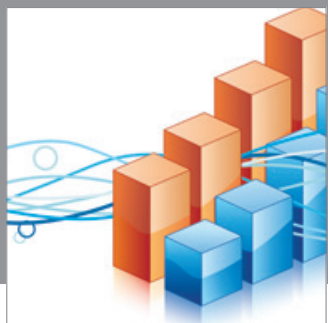

Advances in

Operations Research

mansans

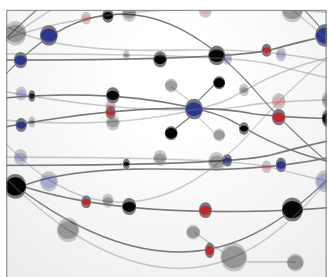

The Scientific World Journal
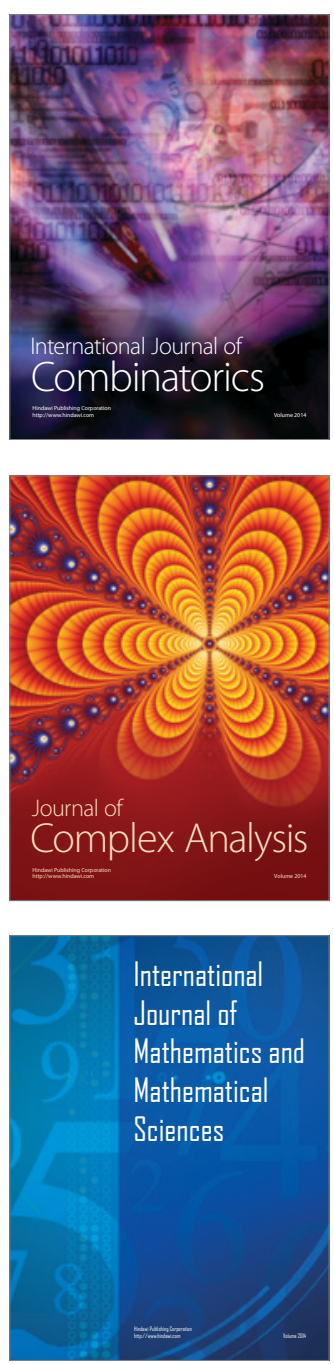
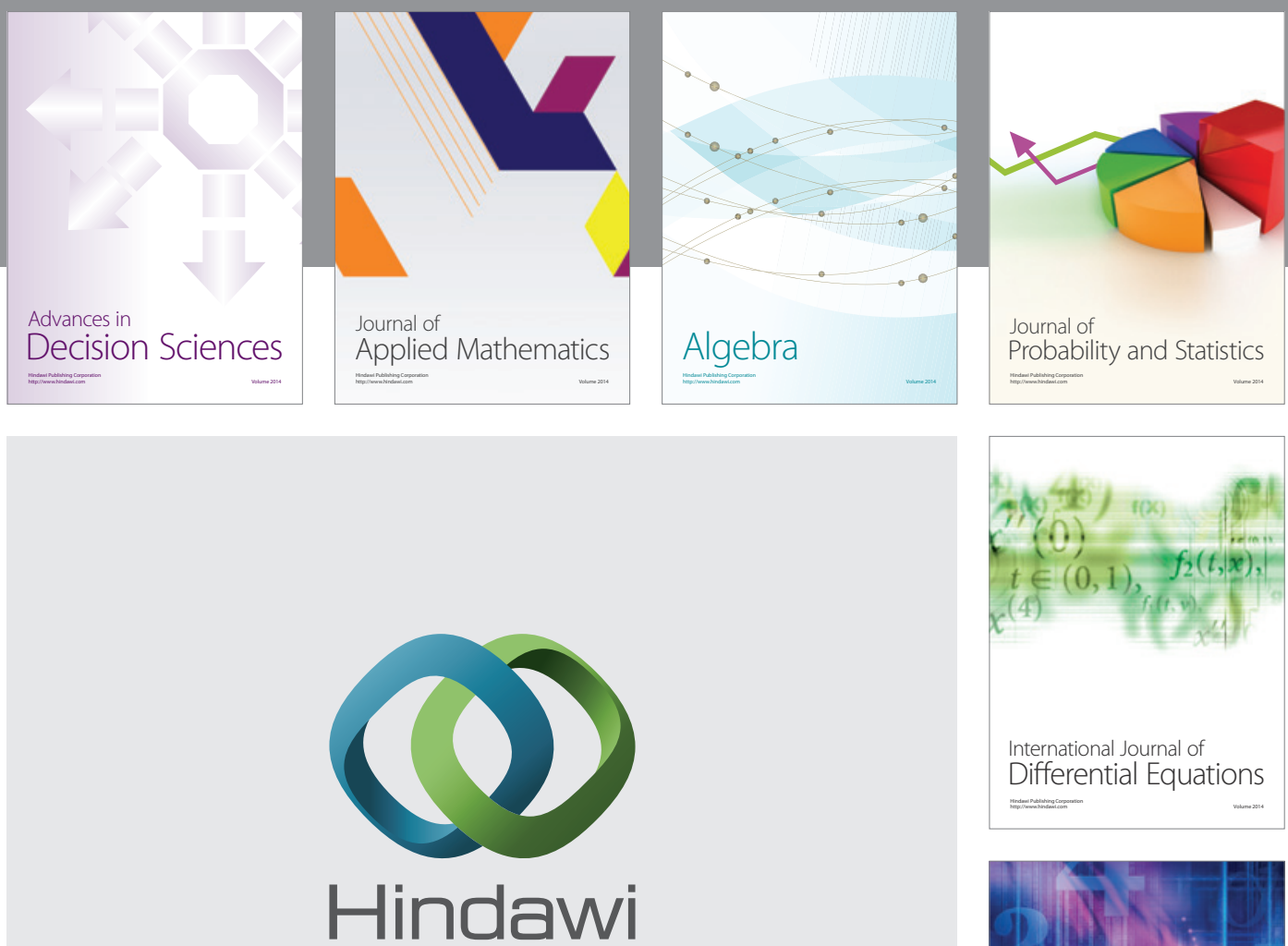

Submit your manuscripts at http://www.hindawi.com
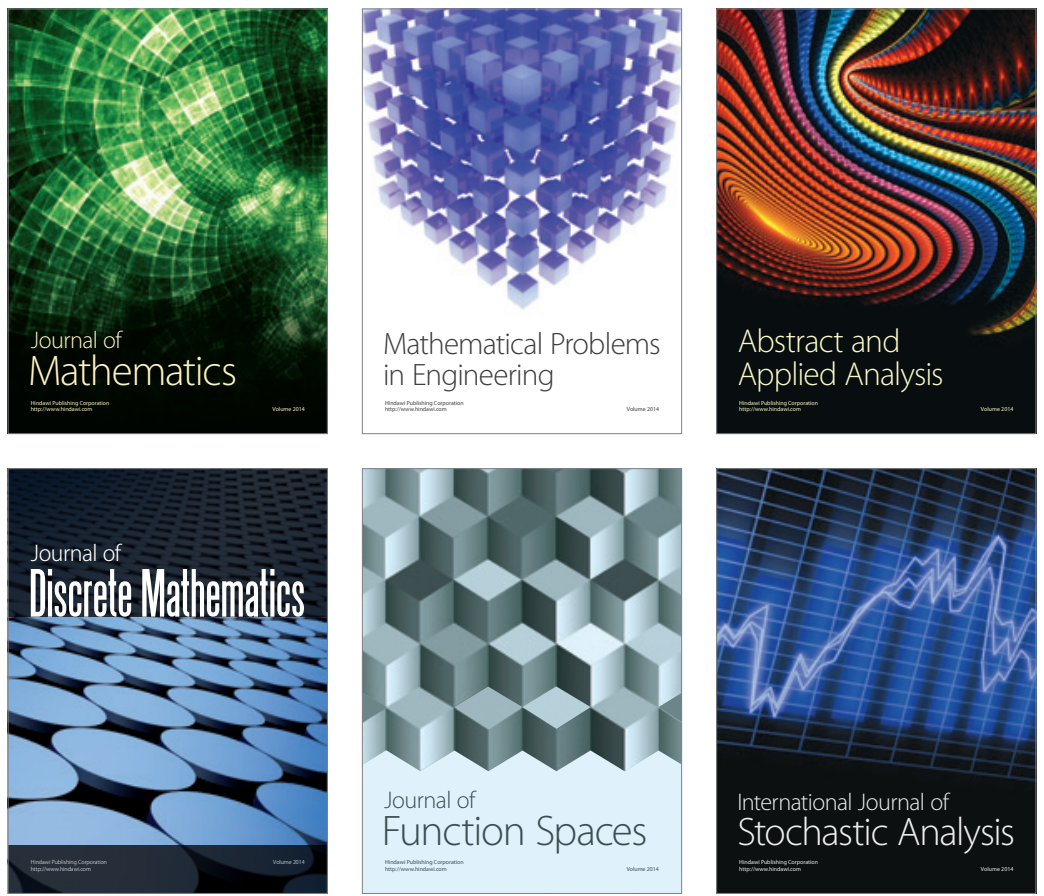

Journal of

Function Spaces

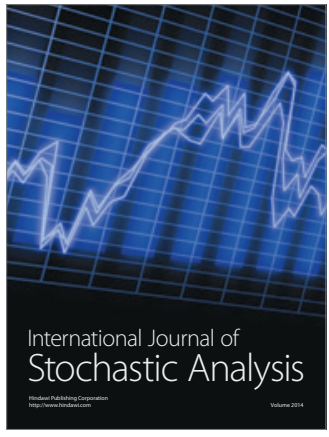

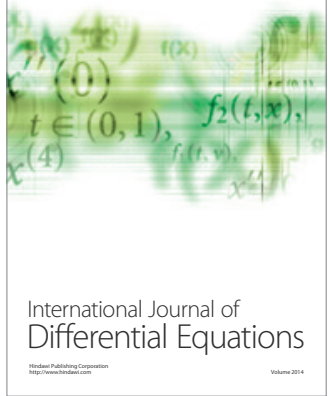
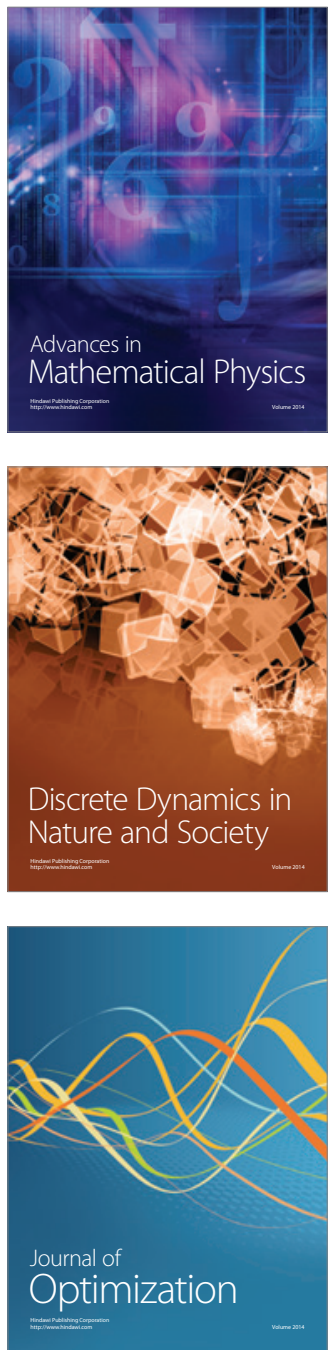